\title{
The collaborative assessment and management of suicide (CAMS): an important model for mental health services to consider
}

\author{
E. Galavan* \\ Clinical Psychology, HSE Dublin North East, Adult Mental Health, St Francis Center, Dublin, Ireland
}

\begin{abstract}
The collaborative assessment and management of suicide (CAMS) model has been developed over 25 years providing mental health clinicians with a flexible, evidenced-based, collaborative and ethically informed way of addressing suicidality with service users. This paper briefly overviews the CAMS model and its advantages and advocates for its use in mental health services both nationally and internationally.
\end{abstract}

Received 11 July 2016; Revised 15 November 2016; Accepted 22 December 2016; First published online 27 February 2017

Data from 122743 self-harm presentations to hospital in Ireland from 2003 to 2013 held by the National Registry of Deliberate Self-Harm showed that the rate of use of highly lethal methods of self-harm has increased significantly since 2004, with the greatest increase among those aged 15-29 years. Many of these individuals will be referred to secondary mental health care and suicidality is an increasingly common presenting issue. Managing suicidality within mental health care is challenging: risk assessment is an inexact science, suicidal behaviour is extremely difficult to predict, causes of suicidality are multifaceted, medications and hospitalisation are rarely effective or sufficient, and sometimes make things worse. Research has shown that suicide risk increases post discharge from inpatient psychiatric care (Bostwick \& Pankratz, 2000; Qin \& Nordentoft, 2005; Meehan et al. 2006). As Shneidman, the father of modern suicidology noted, 'Suicide is chiefly a drama of the mind' (1998). Positive risk taking is essential in dealing effectively with suicidality and yet we work in systems that promote a risk adverse response. Medico-legal concerns are heightened and the question 'should we admit?' frequently dominates the decision-making landscape.

As such a specialised model, or framework, within which we can navigate our interactions, decisions and treatment planning for suicidal service users, becomes compelling. In particular we need a model that has been trialled in the medico-legal realm, is deemed to promote ethically minded decisions, and has an

* Address for correspondence: E. Galavan, HSE Dublin North East, Clinical Psychology, Adult Mental Health, St Francis Center, Station Road, Raheny, Dublin 5, Ireland.

(Email: eoin.galavan@hse.ie) evidence base for its effectiveness. In other words a model that allows a clinician to reasonably assert that the most ethical, evidenced-based, collaborative, recovery-oriented, legally correct, suicide-specific assessment and intervention, was devised for this suicidal person. The collaborative assessment and management of suicide (CAMS) has been developed, trialled and researched over the last 25 years to offer such a model (Jobes, 2016). A full outline of the model, its philosophy and procedures has been outlined (Jobes, 2009).

The CAMS is not a new type of therapy, rather is a psychotherapeutically informed treatment framework that can be wrapped around pre-existing mental health interventions of all types. The primary qualities that differentiate it from other risk assessments or interventions are as follows:

1. CAMS is suicide specific: the CAMS model helps us to arrange mental health interventions in line with what is most pertinent to each individual's suicidality, regardless of diagnosis. We do this by identifying key drivers linked to each individual's suicidality, which become apparent in using the Suicide Status Form, the central assessment tool within the CAMS.

2. The CAMS model directly links risk assessment to interventions. Most risk assessments are somewhat arbitrary elements of routine clinical administration and frequently do little to inform interventions. The CAMS uses risk assessment to guide interventions towards those issues most salient to an individual's suicidal risk.

3. CAMS is collaborative. We do the CAMS with the service user. It is their treatment plan, jointly created, in a collaborative manner. We work 
together to discover what is driving your suicidality; then we work together to figure out what to do about it; and then we work on those problems until suicide is no longer an option for you.

4. Finally, the use of CAMS involves working with the suicidal person and actively tracks suicide risk until it is sufficiently resolved.

\section{Length of time in treatment}

The length of time in treatment until resolution of suicidality has been found to be approximately six to eight sessions (typically lasting between 30-50 minutes each) (Jobes, 2016). Suicidal risk is resolvable in a relatively short period of time with most people, if we dedicate the time to resolving it in a driver-specific fashion. Short, 'one off' or infrequent meetings are unlikely to be sufficient to work with someone through a suicidal crisis to a point of resolution of those issues driving their suicidality. Practice should reflect this reality.

\section{Evidence base}

The evidence base for the CAMS model in terms of its effectiveness is substantial, and growing. There are seven published correlational and open trial studies, and two published randomised controlled trials (RCTs) indicating positive and replicated results associated with reductions in psychological pain, stress, agitation, hopeless, self-hate, overall behavioural self-rated suicide risk, emergency department and primary care visits (see Jobes, 2012 for review) and promising RCT data pertaining to self-harm and suicide attempts (Andreasson et al. 2016). A small $(n=32)$ feasibility RCT comparing CAMS with enhanced care as usual found, despite limited statistical power, a number of statistically significant findings including between group differences in suicidal ideation, overall symptom distress and optimism/hope (Comtois et al. 2011). The between groups effects were most significant at the most distal point from start of treatment, that is 12 months, suggesting an enduring impact of the CAMS intervention.

There are also four RCTs in varying stages of completion or start up (see www.cams-care.com for a full review of research). The CAMS has fared well in a recently published randomised controlled superiority trial examining its effectiveness in comparison with a modified version of dialectical behaviour therapy (DBT) (Andreasson et al. 2016). While there were some methodological and other limits to this study (the DBT arm was not a full course of DBT, training was not as substantial as usual in the DBT arm, the criteria for inclusion were partial rather than full criteria for BPD), no statistically different results were seen between
CAMS and DBT. Inferences about the effectiveness of CAMS in treating BPD are not implied, rather in addressing suicidality in this group. This was an important preliminary study in that DBT has a proven evidence base for reducing self-harm and suicide attempt behaviour. Interestingly, CAMS was effective as DBT in this trial even though patients received fewer sessions in the CAMS arm and a much less intensive form of care. However, replication of this comparison with the above limitations addressed is required before firm conclusions can be drawn about the relative effectiveness of CAMS as compared with DBT.

While DBT is a superb and proven treatment (Linehan et al. 2006), it does have some drawbacks when compared with CAMS. First, the training time involved for clinicians is far more substantial than learning to use CAMS. The CAMS relies on pre-existing skill sets and interventions, shaped in a suicide specific way, as opposed to learning a whole new range of complex theories and interventions from the ground up. DBT is a complex and advanced approach to working with people that is difficult to deliver and hard to maintain within services. DBT also serves a far smaller number of people than that with the equivalent time spent by clinicians using CAMS. One way of thinking about this is as a continuum of complexity of interventions: Applied Suicide Intervention Skills Training (ASIST) or STORM are at one end offering 'once off' models of intervention (with limited empirical support), with little expectation of follow-up, that essentially manage risk and redirect people to further interventions (typically not suicide specific). These models are frankly insufficient to address the needs of most suicidal people, are not treatment frameworks or driver-specific interventions, do not track risk over time and have limited to no evidence base pertaining to their effectiveness in addressing suicidality. DBT, at the other end of the continuum, offers an effective, intensive, resource heavy intervention that is only accessible by a small number of suicidal service users. DBT is arguably most suitable for the most chronically suicidal and complex of presentations (although the comparison study noted above places a question mark over this assumption), and has a substantive evidence base for addressing suicidality and self-harm, particularly with those people categorised as borderline personality disorder. It is not possible or necessary, however, to deliver DBT to all suicidal people in the mental health service. As such CAMS is suitable for the highly populated middle ground between one off interventions like STORM or ASIST, and the intensive resource heavy DBT programme. As a standard service approach to addressing suicidality in mental health care, the CAMS model is the more user-friendly model and is most applicable to most suicidal service users. 


\section{Challenges in implementation}

There are two major challenges in implementing the CAMS; the first is the requirement to develop new skills and understandings, the second is to change the nature of routine practice.

\section{New learning}

The CAMS model requires clinicians learning new skills and a new approach to suicidality. The learning curve in applying the CAMS involves adopting a particular philosophy of care (collaborative and psychotherapeutically informed), consolidation of previously learned skills in a suicide specific context, and mastering multiple modes of intervening with, assessing and managing risk in a coherent relationshipbased model (Jobes, 2016).

\section{Time required and change in practice routine}

Time is required to do all mental health work well. Working with suicidal people typically involves weekly meetings, over a relatively short period of time. Currently, many clinicians dedicate time to working with suicidal people, for example, managing recurring suicidal crises, with little or no focus on resolving drivers and suicidality in the longer term. It is also unusual for many clinicians to meet with suicidal people on a weekly or regular basis dedicated to resolving those issues driving their suicidality. The changes in practice required are eminently achievable and it is time well spent with rates of resolution of suicidality in the CAMS treated group being at $80 \%$ within 12 sessions (Jobes, 2012, 2016).

\section{It is already working}

The North Dublin Suicide Assessment and Treatment Service (SATS) was established in 2013 by the Clinical Psychology service (Galavan \& Horgan, 2015). SATS is run by Clinical Psychologists connected to Community Mental Health Team across the catchment. Each psychologist dedicates $\sim 2$ hourly slots/week to working with suicidal people using the CAMS and so far the service has had $\sim 100$ service users attend. Unpublished audit suggests promising results consistent with previous research evaluating the CAMS model (Jobes, 2012) (audit data currently submitted for publication). The vast majority of people resolve suicidality in a timely fashion, and there is very little need for hospitalisation.

\section{Advantages to using CAMS}

There are several advantages to using the CAMS. Risk assessment is a necessary element of working with suicidal people. The CAMS provides a frame to document a thorough risk assessment in a short period of time that directly informs treatment. This allows us to focus as much on the issues driving the individual's suicidality, and consider possible interventions, as we do to the task of risk assessment and management. The CAMS is an excellent example of risk assessment made useful and directly applied to a suicide-specific treatment.

The CAMS can be used by all mental health professionals. It is also transferable across clinical environments: for example, it can be started in the inpatient unit, and follow the service user to the community team, or an external agency. Even if the receiving clinician or agency does not have direct training or understanding of the CAMS, the information contained in the documentation is easily understood and extremely valuable. A person can, for example, be discharged from an inpatient unit, with a suicide-specific treatment plan in place, providing information on which issues are most relevant for each individual's suicidality. The CAMS can facilitate decision making regarding discharge in the context of ongoing suicidal risk that is mild or moderate in nature, with safety planning, and driverspecific interventions set in motion. In the context of very high risk cases the CAMS provides a focus for hospital-based interventions to be driver and suicide specific, regardless of diagnostic category, with a view towards discharge as risk decreases over time. The vast majority of suicidal service users do not require an inpatient stay to address their suicidal risk [keeping in mind that inpatient stays are associated with increasing suicide risk (Large \& Ryan, 2014]. The CAMS provides a coherent, evidenced-based treatment frame within which to navigate and document this decision making.

Training in the CAMS model has been delivered to staff from all disciplines in North Dublin Adult Mental Health and the College of Psychiatry in Ireland, with very positive feedback on all occasions, from hundreds of mental health clinicians and therapists in Ireland, and many more in the United States. CAMS training is available in Ireland and the United Kingdom (see www.camscare.com).

\section{Acknowledgement}

None.

\section{Financial Support}

This research received no specific grant from any funding agency, commercial or not-for-profit sectors. 


\section{Conflicts of Interest}

Dr Galavan is a senior CAMS-care senior consultant and provides training in this model. No payments were received in authoring this article.

\section{Ethical Standards}

The author asserts that all procedures contributing to this work comply with the ethical standards of the relevant national and institutional committee on human experimentation with the Helsinki Declaration of 1975 , as revised in 2008. The author asserts that ethical approval for publication was not required by their local REC.

\section{References}

Andreasson K, Krogh J, Wenneberg C, Jessen HKL, Krakauer K, Gluud C, et al. (2016). Effectiveness of dialectical behaviour therapy versus collaborative assessment and management of suicidality treatment for reduction of self-harm in adults with borderline personality traits and disorder-a randomized observer-blinded clinical trial. Depression and Anxiety 0, 1-11.

Bostwick JM, Pankratz VS (2000). Affective disorders and suicide risk: A re-examination. American Journal of Psychiatry 157, 1925-1932.

Comtois KA, Jobes DA, O'Connor S, Atkins DC, Janis K, Chessen C, Landes SJ, Holen A, Youdelis-Flores C (2011). Collaborative assessment and management of suicidality
(CAMS): feasibility trial for next day appointment services. Depression and Anxiety 28, 963-972.

Galavan E, Horgan H (2015). Developing a suicide assessment and treatment service based on the collaborative assessment and management of suicide. Irish Psychologist 41, 171-173.

Jobes DA (2009). The CAMS approach to suicide risk: philosophy and clinical procedures. Suicidologi 14, 3-7.

Jobes DA (2012). The collaborative assessment and management of suicidality (CAMS): an evolving evidencebased clinical approach to suicidal risk. Suicide Life Threatening Behaviour 42, 640-653.

Jobes DA (2016). Managing Suicidal Risk: A Collaborative Approach, 2nd edn. Guilford Press: New York.

Large M, Ryan CJ (2014). Disturbing findings about the risk of suicide and psychiatric hospitals. Social Psychiatry and Psychiatric Epidemiology 49, 1353-1355.

Linehan MM, Comtois KA, Murray AM, Brown MZ, Gallop RJ, Hear HL, et al. (2006). Two year randomised control trial and follow up of dialectical behaviour therapy verses therapy by experts for suicidal behaviours and borderline personality disorder. Archives of General Psychiatry 63, 757-766.

Meehan J, Kapur N, Hunt IM, Turnbull P, Robinson J, Bickley H, et al. (2006). Suicide in mental health in-patients and within 3 months of discharge: national clinical survey. British Journal of Psychiatry 188, 129-134.

Qin P, Nordentoft M (2005). Suicide risk in relation to psychiatric hospitalisation: evidence based on longitudinal registers. Archives of General Psychiatry 62, 427-432.

Shneidman ES (1998). The Suicidal Mind. Aronson: Northfield, NJ. 